\title{
Serum Anion Gap Is Associated with All-Cause Mortality among Critically Ill Patients with Congestive Heart Failure
}

\author{
Yiyang Tang $\oplus^{1},{ }^{1}$ Wenchao Lin, ${ }^{1}$ Lihuang Zha, ${ }^{1}$ Xiaofang Zeng, ${ }^{1}$ Xiaoman Zeng, ${ }^{2}$ Guojun Li, ${ }^{3}$ \\ Zhenghui Liu, ${ }^{4}$ and Zaixin Yu ${ }^{10}{ }^{1,5}$ \\ ${ }^{1}$ Department of Cardiology, Xiangya Hospital, Central South University, Changsha, Hunan, China \\ ${ }^{2}$ Department of Cardiology, Wenchang People's Hospital, Wenchang, Hainan, China \\ ${ }^{3}$ Department of Emergency, The Third Hospital of Changsha, Changsha, Hunan, China \\ ${ }^{4}$ Department of Neurology, Xiangya Hospital, Central South University, Changsha, Hunan, China \\ ${ }^{5}$ National Clinical Research Center for Geriatric Disorders (Xiangya), Changsha, Hunan, China \\ Correspondence should be addressed to Zaixin Yu; yuzaixin@126.com
}

Received 19 August 2020; Revised 14 October 2020; Accepted 28 October 2020; Published 18 November 2020

Academic Editor: Heng Zhou

Copyright (C) 2020 Yiyang Tang et al. This is an open access article distributed under the Creative Commons Attribution License, which permits unrestricted use, distribution, and reproduction in any medium, provided the original work is properly cited.

Background. Congestive heart failure (CHF) is a complex clinical syndrome, with high morbidity and mortality. Serum anion gap (SAG) is associated with the severity of various cardiovascular diseases. However, the role of SAG indicators in CHF is unclear. Methods and Results. A retrospective analysis of data from Multiparameter Intelligent Monitoring in Intensive Care III version 1.4 was conducted in critically ill patients with CHF. The clinical information of each patient, including demographic data, comorbidities, vital signs, scores, and laboratory indicators, were successfully obtained. Cox proportional hazards models were used to determine the relationship between SAG and mortality in patients with CHF, the consistency of which was further verified by subgroup analysis. Results. A total of 7426 subjects met the inclusion criteria. Multivariate analysis showed that after adjusting for age, gender, ethnicity, and other potential confounders, increased SAG was significantly related to an increase in 30- and 90-day all-cause mortalities of critically ill patients with CHF compared with decreased SAG (tertile 3 versus tertile 1: adjusted hazard ratio, 95\% confidence interval: 1.74, 1.46-2.08; 1.53, 1.32-1.77). Subgroup analysis indicated that the association between SAG and all-cause mortality presented similarities in most strata. Conclusion. SAG at admission could be a promising predictor of all-cause mortality in critically ill patients with CHF.

\section{Introduction}

Congestive heart failure (CHF) is the end-stage manifestation of various cardiovascular diseases with structural and functional disruptions in the myocardium, causing restricted ventricular ejection or filling, systemic blood circulation disorder, insufficient perfusion of tissues and organs, and ultimate death [1]. CHF has become a major public health problem with more than 23 million patients affected worldwide, and it still has a high mortality despite great progress in diagnosis and treatment for the last few decades [2]. Thus, how to early identify high-risk patients, determine their prognosis, and formulate effective individualized interventions have attracted growing attention from clinicians in recent years.
Metabolic acidosis is a common complication of CHF; it is closely related to ischemia and hypoxia of tissue caused by hemodynamic disorders and the use of diuretics [3]. In turn, acidosis and accompanying hyperkalemia could further weaken myocardial contractility, creating a vicious circle. Acidosis serves as the independent predictor for the longterm prognosis of $\mathrm{CHF}$, and $\mathrm{pH}$ value could help to stratify the risks [4]. However, the predictive value of other laboratory parameters that reflect the acid-base imbalance in CHF requires more evidence, such as serum anion gap (SAG).

SAG refers to the difference between undetermined anions and cations. It indicates the concentration of fixed acids in plasma, and it is a commonly used and easily obtained laboratory parameter of acid-base imbalance [5]. Some recent studies have confirmed that SAG is elevated 
and closely associated with poor prognosis of various diseases, including acute pesticide poisoning [6], sepsis [7], acute and chronic kidney injury [8,9], trauma [10], and coronary artery disease [11]. However, whether the anion gap could be used as a prognostic marker for critically ill patients with CHF remains unclear. Therefore, the present study is aimed at investigating the association between SAG and mortality in these patients.

\section{Materials and Methods}

2.1. Data Source. The Multiparameter Intelligent Monitoring in Intensive Care III version 1.4 (MIMIC-III v1.4) is a freely accessible critical care database developed and operated by the Massachusetts Institute of Technology; it contains detailed clinical data of 53,423 adult patients (age more than 16 years) from June 2001 to October 2012 in the intensive care units (ICUs) of Beth Israel Deaconess Medical Center [12]. Before implementing the present research, author Tang completed and passed the CITI "Data or Specimens Only Research" course (No. 9014457) and obtained authorization for database access. In need of special note here is that this database was approved by the Institutional Review Boards of Massachusetts Institute of Technology (Cambridge, MA, USA) and Beth Israel Deaconess Medical Center (Boston, MA, USA), and no additional ethical approval needed to be provided.

2.2. Study Population and Design. In the MIMIC-III database, all adult patients aged over 18 years old, first admitted to the ICU, and diagnosed with CHF were included in the study in accordance with the ninth revision of the International Classification of Diseases $(I C D)$ code (code $=428.0)$. Patients with any of the following criteria were excluded from the study: (1) ICU stay time of less than 24 hours, (2) no anion gap results within 24 hours of admission to the ICU, and (3) survival time of less than 0 (some organ donors may die earlier than admission). The workflow is shown in Figure 1.

The starting point of the study was defined as the time of admission to the ICU, and the endpoint was the time of death, 30 , or 90 days after admission. The patients' 30- and 90-day mortalities were chosen as the primary outcome of interest, and death data were extracted from the Social Security Death Index. The secondary outcomes were the readmission rate and the composite outcome of major adverse cardiac events (MACEs), including all-cause mortality, hospitalization with acute heart failure, heart transplantation, and mechanical circulatory support [13].

2.3. Data Extraction and Preparation. Structured query language (SQL) was utilized to extract clinical data with PostgreSQL tools (version 9.6), including demographics, comorbidities, vital signs, severity scores, laboratory tests, and interventions. Demographics included age, gender, and ethnicity. For the privacy protection of patients, the database has had the date of birth shifted to exactly 300 years for patients older than 89 years. Before the analysis, the age of these patients was adjusted in accordance with the following formula: real age $=$ age $-300+89$ [14]. Vital

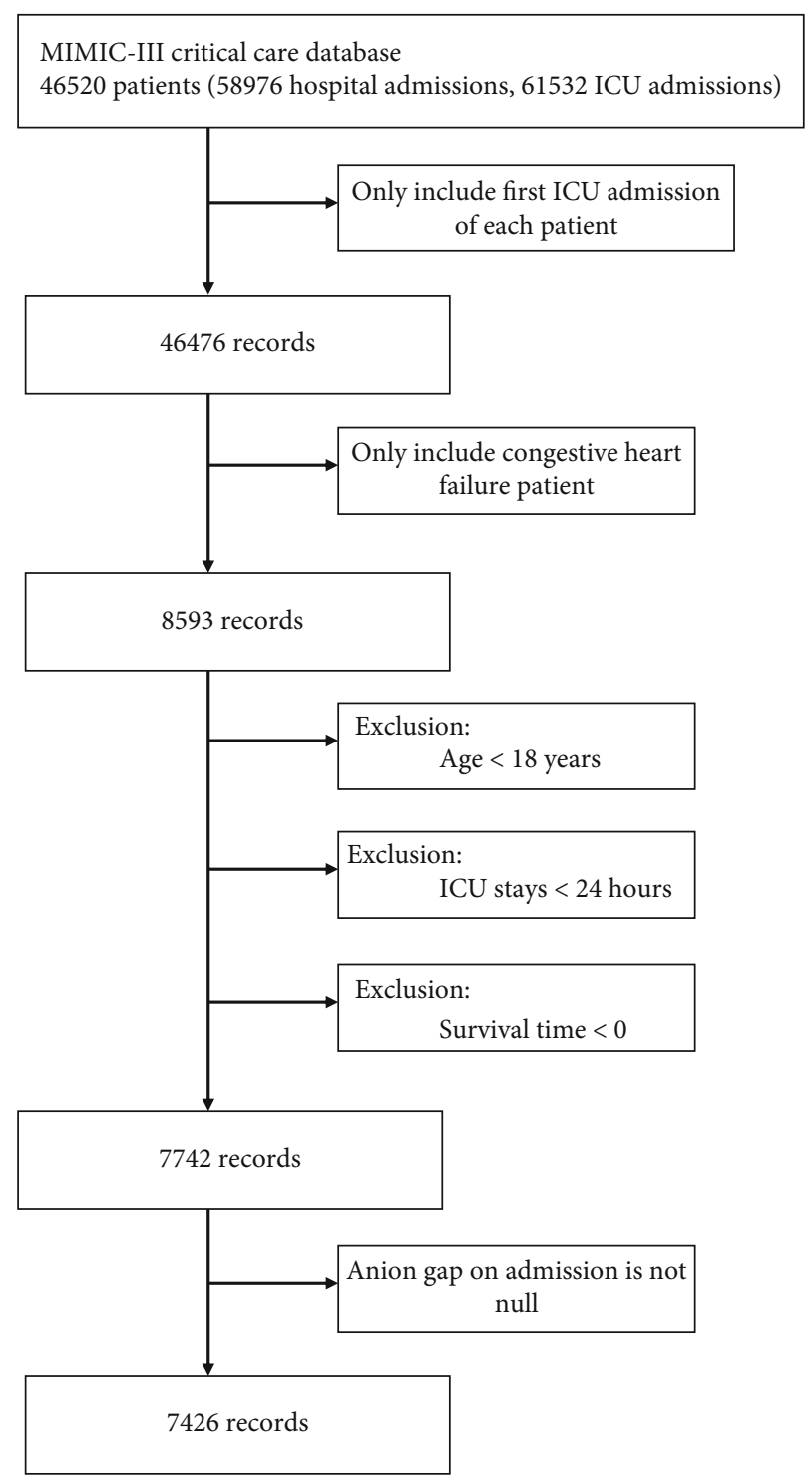

FIgURE 1: Workflow of the data extraction. The inclusion and exclusion criteria of the study subjects were revealed, and 7426 subjects were finally included. ICU: intensive care unit.

signs consisted of temperature, respiratory rate (RR), heart rate (HR), systolic blood pressure (SBP), diastolic blood pressure (DBP), mean blood pressure (MBP), and percutaneous oxygen saturation $\left(\mathrm{SpO}_{2}\right)$. Comorbidities included acute myocardial infarction (AMI), atrial fibrillation, valvular heart diseases, pulmonary circulation diseases, hypertension (HBP), diabetes, pneumonia, respiratory failure, liver disease, renal failure, stroke, and malignancy. The sequential organ failure assessment (SOFA) score and the simplified acute physiology score II (SAPSII) were calculated for each patient when entering the ICU to assess the severity of the disease $[15,16]$. Laboratory tests within 24 hours after ICU admission were also extracted, including SAG, white blood cell (WBC), platelet, hemoglobin, blood urea nitrogen (BUN), creatinine, sodium, potassium, chloride, bicarbonate, glucose, prothrombin time (PT), 
TABLE 1: Details of missing values.

\begin{tabular}{|c|c|c|c|c|c|}
\hline Variables & $\begin{array}{c}\text { The number of missing } \\
\text { values }\end{array}$ & $\begin{array}{c}\text { The percent of missing } \\
\text { values }\end{array}$ & Variables & $\begin{array}{c}\text { The number of missing } \\
\text { values }\end{array}$ & $\begin{array}{c}\text { The percent of missing } \\
\text { values }\end{array}$ \\
\hline SBP & 39 & $0.5 \%$ & Glucose & 48 & $0.6 \%$ \\
\hline DBP & 39 & $0.5 \%$ & Lactate & 1993 & $26.8 \%$ \\
\hline MBP & 32 & $0.4 \%$ & $\begin{array}{c}\text { NT- } \\
\text { proBNP }\end{array}$ & 6231 & $83.9 \%$ \\
\hline $\mathrm{RR}$ & 37 & $0.5 \%$ & PT & 111 & $1.5 \%$ \\
\hline HR & 32 & $0.4 \%$ & APTT & 137 & $1.8 \%$ \\
\hline $\mathrm{SpO}_{2}$ & 34 & $0.5 \%$ & Sodium & 1 & $<0.1 \%$ \\
\hline Temperature & 255 & $3.4 \%$ & Potassium & 1 & $<0.1 \%$ \\
\hline Weight & 484 & $6.5 \%$ & Chloride & 1 & $<0.1 \%$ \\
\hline WBC & 68 & $0.9 \%$ & Bicarbonate & 2 & $<0.1 \%$ \\
\hline Platelet & 65 & $0.8 \%$ & Creatinine & 2 & $<0.1 \%$ \\
\hline Hemoglobin & 88 & $1.1 \%$ & BUN & 4 & $<0.1 \%$ \\
\hline
\end{tabular}

Note: SBP: systolic blood pressure; DBP: diastolic blood pressure; MBP: mean blood pressure; RR: respiratory rate; HR: heart rate; $\mathrm{SpO}_{2}$ : percutaneous oxygen saturation; WBC: white blood cell; BUN: blood urea nitrogen; PT: prothrombin time; APTT: activated partial thromboplastin time; NT-proBNP: N-terminal probrain natriuretic peptide.

activated partial thromboplastin time (APTT), lactate, and plasma N-terminal probrain natriuretic peptide (NTproBNP). Interventions included vasopressor, dialysis, and mechanical ventilation.

After extraction was performed, the raw data were merged and disposed with patient identifiers by using STATA version 16 (https://www.stata.com/). The "winsorize" function was used to reduce the effect of outliers, and multivariate multiple imputation with chained equations was utilized to impute the missing values. The details of the missing value are shown in Table 1. More than $20 \%$ of the subjects in this study cohort did not have records of lactate and NT-proBNP, which was converted and considered as a dummy variable in the models to avoid possible bias caused by direct filling of missing values [17].

2.4. Statistical Analysis. The baseline data of all subjects were divided into three groups by SAG tertiles. Continuous variables were presented as mean \pm SD or medians and interquartile range. Kruskal-Wallis test was used to conduct a hypothesis test for continuous variables. Categorical variables were expressed by numbers and percentages, which were analyzed using Chi-square (or Fisher's exact) tests. A generalized additive model (GAM) was used to determine the nonlinear association between SAG and 30-day all-cause mortalities in critically ill patients with CHF. In addition, the relationship between SAG and the survival in patients with HF was visually shown through the Kaplan-Meier $(\mathrm{K}-\mathrm{M})$ curve and tested using the log-rank test.

Cox proportional hazard model was used to analyze the relationship between SAG and 30-day and 90-day all-cause mortality in critically ill patients with $\mathrm{HF}$ and determine the independent prognostic value of SAG in these patients, with the first tertile or quartile as the reference and adjusting for potential confounders. Two multivariate models were conducted, and the results were described as hazard ratio (HR) with $95 \%$ confidence intervals (CIs). In model I, the covariates were only adjusted for the confounders' age, sex, and ethnicity. On the basis of model I, model II was further adjusted for other confounders, including temperature, HR, RR, SBP, $\mathrm{DBP}, \mathrm{SpO}_{2}$, weight, atrial fibrillation, liver disease, valvular heart diseases, pulmonary circulation diseases, pneumonia, respiratory failure, diabetes, stroke, malignancy, SOFA, SAPSII, lactate, NT-proBNP, PT, WBC, BUN, creatinine, potassium, bicarbonate, glucose, vasopressor, dialysis, and mechanical ventilation. These factors were chosen as confounders on the basis of their association with the outcomes or a change in effect estimate exceeding 10\% [18]. Variance inflation factor was used to test the collinearity between variables with 5 as the threshold, and the variable MBP and chloride were deleted. The associations between SAG and readmission rate and MACEs were determined using multivariate logistic regression with results expressed as the odds ratio (OR) with 95\% CIs. Besides, a subgroup analysis of the correlation between SAG and 30-day all-cause mortality was performed to examine whether the effect of SAG in various subgroups differed. All statistical analyses were performed on EmpowerStats version 2.20 (http://www .empowerstats.com/cn/, X\&Y solutions, Inc., Boston, MA) and $\mathrm{R}$ software version 3.4.3; $P<0.05$ (two-sided) was considered statistically significant.

\section{Results}

3.1. Clinical Characteristics of Subjects. A total of 7426 subjects were included in this retrospective study, 3971 of whom were male and 3455 were female. The age of the subjects was generally high, with a median of 75.3 years old. The subjects were mostly white, accounting for 73.3 percent share of the total. And the 30- and 90-day overall mortalities were $17.7 \%$ and $24.4 \%$, respectively. The clinical characteristics of these subjects stratified by SAG tertiles are shown in Table 2. A total of 2334 subjects were in the low-SAG group (tertile $1, \mathrm{SAG}<13$ ), 2519 subjects 
TABLE 2: The clinical characteristics of critically ill patients with CHF according to SAG levels.

\begin{tabular}{|c|c|c|c|c|}
\hline Characteristics & $<13(n=2334)$ & $\begin{array}{l}\text { rum anion gap (mmol } \\
\geq 13,<16(n=2519)\end{array}$ & $\geq 16(n=2573)$ & $P$ value \\
\hline Age (years) & $72.2 \pm 13.2$ & $73.2 \pm 13.2$ & $72.5 \pm 13.5$ & 0.013 \\
\hline Gender, $n(\%)$ & & & & 0.278 \\
\hline Male & $1280(54.8)$ & $1332(52.9)$ & $1359(52.8)$ & \\
\hline Female & $1054(45.2)$ & $1187(47.1)$ & $1214(47.2)$ & \\
\hline Ethnicity, $n(\%)$ & & & & 0.002 \\
\hline White & $1744(74.7)$ & $1881(74.7)$ & $1821(70.8)$ & \\
\hline Black & $152(6.5)$ & $187(7.4)$ & $230(8.9)$ & \\
\hline Other & $438(18.8)$ & $451(17.9)$ & $522(20.3)$ & \\
\hline $\mathrm{SBP}, \mathrm{mmHg}$ & $116.7 \pm 15.2$ & $117.6 \pm 17.0$ & $116.2 \pm 17.8$ & 0.004 \\
\hline $\mathrm{DBP}, \mathrm{mmHg}$ & $57.3 \pm 9.4$ & $58.0 \pm 10.4$ & $58.1 \pm 10.9$ & 0.013 \\
\hline $\mathrm{MBP}, \mathrm{mmHg}$ & $75.3 \pm 9.7$ & $76.1 \pm 10.5$ & $75.9 \pm 11.1$ & 0.038 \\
\hline $\mathrm{HR}$, beats/minute & $84.0 \pm 14.2$ & $84.0 \pm 15.6$ & $86.8 \pm 16.7$ & $<0.001$ \\
\hline $\mathrm{RR}$, beats/minute & $18.7 \pm 3.7$ & $19.6 \pm 3.9$ & $20.2 \pm 4.3$ & $<0.001$ \\
\hline Temperature, ${ }^{\circ} \mathrm{C}$ & $36.8 \pm 0.6$ & $36.8 \pm 0.6$ & $36.7 \pm 0.7$ & $<0.001$ \\
\hline $\mathrm{SpO}_{2}, \%$ & $97.3 \pm 1.9$ & $96.9 \pm 2.0$ & $96.8 \pm 2.4$ & $<0.001$ \\
\hline Weight, kg & $81.9 \pm 24.1$ & $81.2 \pm 24.3$ & $81.3 \pm 24.8$ & 0.212 \\
\hline \multicolumn{5}{|l|}{ Scoring systems } \\
\hline SOFA & $4.5 \pm 2.7$ & $4.4 \pm 2.8$ & $5.7 \pm 3.3$ & $<0.001$ \\
\hline SAPSII & $37.1 \pm 11.6$ & $38.6 \pm 12.6$ & $44.2 \pm 14.0$ & $<0.001$ \\
\hline Dialysis, $n(\%)$ & $60(2.6)$ & $115(4.6)$ & $440(17.1)$ & $<0.001$ \\
\hline Vasopressor, $n(\%)$ & $558(23.9)$ & $498(19.8)$ & $666(25.9)$ & $<0.001$ \\
\hline Ventilation, $n(\%)$ & $624(26.7)$ & $784(31.1)$ & $1000(38.9)$ & $<0.001$ \\
\hline ICU LOS, hours & $116.7 \pm 149.8$ & $130.3 \pm 164.5$ & $147.4 \pm 180.9$ & $<0.001$ \\
\hline 30-day mortality, $n(\%)$ & $254(10.9)$ & $399(15.8)$ & $665(25.8)$ & $<0.001$ \\
\hline 90-day mortality, $n(\%)$ & $411(17.6)$ & $579(23.0)$ & $882(34.3)$ & $<0.001$ \\
\hline \multicolumn{5}{|l|}{ Comorbidities, n (\%) } \\
\hline Liver diseases & $97(4.2)$ & $89(3.5)$ & $119(4.6)$ & 0.144 \\
\hline Renal failure & $325(13.9)$ & $552(21.9)$ & $813(31.6)$ & $<0.001$ \\
\hline Atrial fibrillation & $1053(45.1)$ & $1134(45.0)$ & $1116(43.4)$ & 0.377 \\
\hline Stroke & $105(4.5)$ & $152(6.0)$ & $134(5.2)$ & 0.056 \\
\hline AMI & $121(5.2)$ & $207(8.2)$ & $270(10.5)$ & $<0.001$ \\
\hline VHD & $178(7.6)$ & $248(9.8)$ & $264(10.3)$ & 0.003 \\
\hline PCD & $130(5.6)$ & $140(5.6)$ & $139(5.4)$ & 0.959 \\
\hline HBP & $278(11.9)$ & $467(18.5)$ & $673(26.2)$ & $<0.001$ \\
\hline Diabetes & $786(33.7)$ & $846(33.6)$ & $1021(39.7)$ & $<0.001$ \\
\hline Pneumonia & $440(18.9)$ & $581(23.1)$ & $640(24.9)$ & $<0.001$ \\
\hline Respiratory failure & 487 (20.9) & $628(24.9)$ & $774(30.1)$ & $<0.001$ \\
\hline Malignancy & $94(4.0)$ & $145(5.8)$ & $118(4.6)$ & 0.015 \\
\hline \multicolumn{5}{|l|}{ Laboratory tests } \\
\hline $\mathrm{WBC}(\mathrm{K} / \mu \mathrm{l})$ & $11.4 \pm 6.2$ & $12.7 \pm 27.8$ & $13.8 \pm 8.3$ & $<0.001$ \\
\hline Platelet $(\mathrm{K} / \mu \mathrm{l})$ & $196.2 \pm 95.4$ & $218.7 \pm 98.2$ & $240.1 \pm 118.0$ & $<0.001$ \\
\hline Hemoglobin (g/dl) & $10.2 \pm 1.9$ & $10.8 \pm 2.0$ & $10.9 \pm 2.1$ & $<0.001$ \\
\hline Creatinine (mg/dl) & $1.0 \pm 0.6$ & $1.4 \pm 0.9$ & $2.4 \pm 2.0$ & $<0.001$ \\
\hline BUN (mg/dl) & $23.2 \pm 13.7$ & $30.0 \pm 19.0$ & $45.1 \pm 28.8$ & $<0.001$ \\
\hline Glucose (mg/dl) & $129.0 \pm 53.7$ & $144.7 \pm 60.9$ & $171.3 \pm 94.8$ & $<0.001$ \\
\hline
\end{tabular}


TABLE 2: Continued.

\begin{tabular}{|c|c|c|c|c|}
\hline Characteristics & $<13(n=2334)$ & $\begin{array}{l}\text { rum anion gap (mmo } \\
\geq 13,<16(n=2519)\end{array}$ & $\geq 16(n=2573)$ & $P$ value \\
\hline Sodium $(\mathrm{mmol} / \mathrm{L})$ & $138.8 \pm 4.6$ & $138.9 \pm 4.6$ & $138.2 \pm 5.4$ & $<0.001$ \\
\hline Potassium (mmol/L) & $4.1 \pm 0.6$ & $4.2 \pm 0.7$ & $4.4 \pm 0.8$ & $<0.001$ \\
\hline Chloride (mmol/L) & $106.2 \pm 6.2$ & $104.6 \pm 5.9$ & $102.7 \pm 6.6$ & $<0.001$ \\
\hline Bicarbonate $(\mathrm{mmol} / \mathrm{L})$ & $26.5 \pm 4.9$ & $24.8 \pm 4.2$ & $21.5 \pm 4.7$ & $<0.001$ \\
\hline PT (second) & $15.9 \pm 7.1$ & $16.7 \pm 9.9$ & $17.8 \pm 12.0$ & $<0.001$ \\
\hline \multicolumn{5}{|l|}{ Laboratory tests } \\
\hline APTT (second) & $38.7 \pm 23.8$ & $38.8 \pm 25.2$ & $40.4 \pm 26.6$ & 0.002 \\
\hline Lactate, $n(\%)$ & & & & $<0.001$ \\
\hline$<1.7 \mathrm{mmol} / \mathrm{L}$ & $1076(46.1)$ & $865(34.3)$ & $694(27.0)$ & \\
\hline$\geq 1.7 \mathrm{mmol} / \mathrm{L}$ & $634(27.2)$ & $858(34.1)$ & $1306(50.8)$ & \\
\hline No test & $624(26.7)$ & $796(31.6)$ & $573(22.3)$ & \\
\hline NT-proBNP, $n(\%)$ & & & & $<0.001$ \\
\hline$<4983 \mathrm{pg} / \mathrm{ml}$ & $192(8.2)$ & $219(8.7)$ & $186(7.2)$ & \\
\hline$\geq 4983 \mathrm{pg} / \mathrm{ml}$ & $118(5.1)$ & $200(7.9)$ & $280(10.9)$ & \\
\hline No test & $2024(86.7)$ & $2100(83.4)$ & $2107(81.9)$ & \\
\hline
\end{tabular}

Note: CHF: congestive heart failure; SAG: serum anion gap; SBP: systolic blood pressure; DBP: diastolic blood pressure; MBP: mean blood pressure; RR: respiratory rate; HR: heart rate; $\mathrm{SpO}_{2}$ : percutaneous oxygen saturation; SOFA: stroke, and malignancy. Calculate the sequential organ failure assessment score; SAPSII: simplified acute physiology score II; ICU: intensive care unit; LOS: length of stay; AMI: acute myocardial infarction; VHD: valvular heart diseases; PCD: pulmonary circulation diseases; HBP: hypertension; WBC: white blood cell; BUN: blood urea nitrogen; PT: prothrombin time; APTT: activated partial thromboplastin time; NT-proBNP: N-terminal probrain natriuretic peptide.

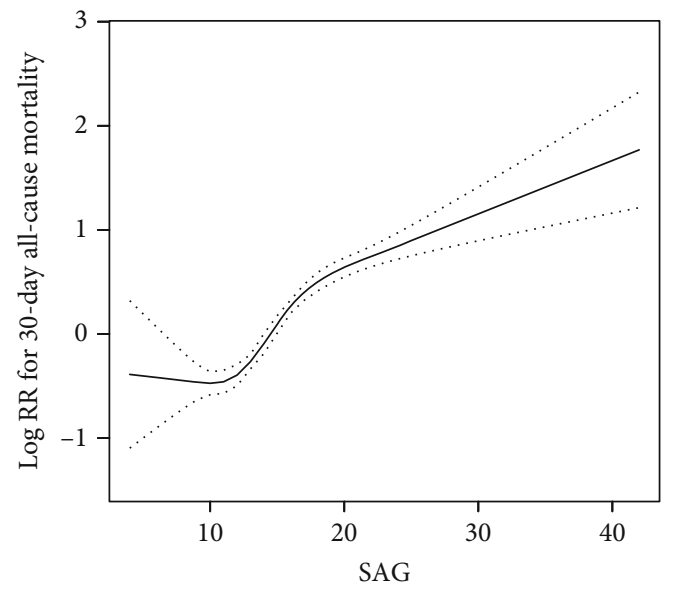

FIGURE 2: Construction of the smooth curve fitting of the risk of 30day all-cause mortality and SAG using a generalized additive model. Dashed curves were for the $95 \%$ of confidence interval.

were in the mid-SAG group (tertile 2, SAG $\geq 13$, and SAG < 16), and 2573 subjects were in the high-SAG group (tertile 3, SAG $\geq 16$ ). Subjects with higher SAG levels had more comorbidities of HBP, AMI, diabetes, renal failure, and respiratory failure, with higher mortality, higher SOFA and SAPSII scores, and higher rates of use of vasopressor, dialysis, and mechanical ventilation.

3.2. Primary Outcome: Association between SAG and AllCause Mortality. As shown in Figure 2, the result of GAM analysis indicated a U-shaped relationship between SAG

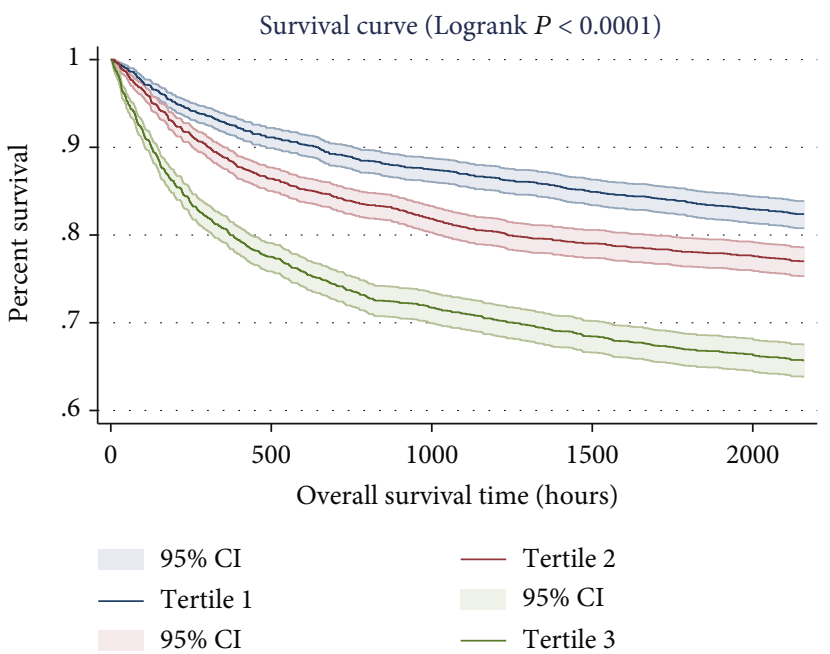

FIgURE 3: Kaplan-Meier survival curves for critically ill patients with CHF based on tertile of SAG. $x$-axis: survival time (hours). $y$ -axis: cumulative survival probability. CHF: congestive heart failure. SAG: serum anion gap.

and 30-day all-cause mortality in critically ill patients with $\mathrm{CHF}$. The K-M survival curve illustrated that subjects with increased SAG levels presented a decreased survival rate and shortened survival time (Log-rank $P<0.0001$, Figure 3 ).

The Cox proportional hazard model was used to assess the association between SAG and all-cause mortality (Table 3). In model I, after adjusting for age, sex, and ethnicity, high levels of SAG were significantly associated with increased risk of 30and 90-day all-cause mortalities (tertile 3 versus tertile 1: HR, 
TABLE 3: HRs (95\% CIs) for all-cause mortality across groups of serum anion gap.

\begin{tabular}{|c|c|c|c|c|c|c|}
\hline \multirow{2}{*}{ Variable } & \multicolumn{2}{|c|}{ Crude } & \multicolumn{2}{|c|}{ Model I } & \multicolumn{2}{|c|}{ Model II } \\
\hline & HR (95\%CIs) & $P$ value & HR (95\%CIs) & $P$ value & HR (95\%CIs) & $P$ value \\
\hline \multicolumn{7}{|c|}{ 30-day all-cause mortality } \\
\hline Anion gap & $1.10(1.09,1.11)$ & $<0.0001$ & $1.11(1.10,1.12)$ & $<0.0001$ & $1.05(1.03,1.07)$ & $<0.0001$ \\
\hline \multicolumn{7}{|c|}{ Anion gap (tertile) } \\
\hline$<13$ & 1 (ref) & & 1 (ref) & & 1 (ref) & \\
\hline$\geq 13,<16$ & $1.50(1.28,1.75)$ & $<0.0001$ & $1.47(1.25,1.72)$ & $<0.0001$ & $1.28(1.09,1.50)$ & 0.0031 \\
\hline$\geq 16$ & $2.63(2.27,3.03)$ & $<0.0001$ & $2.62(2.27,3.03)$ & $<0.0001$ & $1.74(1.46,2.08)$ & $<0.0001$ \\
\hline$P$ for trend & $<0.0001$ & & $<0.0001$ & & $<0.0001$ & \\
\hline \multicolumn{7}{|c|}{ Anion gap (quartile) } \\
\hline$<12$ & 1 (ref) & & 1 (ref) & & 1 (ref) & \\
\hline$\geq 12,<14$ & $1.41(1.15,1.73)$ & 0.0009 & $1.39(1.13,1.70)$ & 0.0016 & $1.22(0.99,1.49)$ & 0.0621 \\
\hline$\geq 14,<17$ & $1.80(1.50,2.17)$ & $<0.0001$ & $1.75(1.46,2.11)$ & $<0.0001$ & $1.53(1.26,1.86)$ & $<0.0001$ \\
\hline$\geq 17$ & $3.16(2.65,3.78)$ & $<0.0001$ & $3.20(2.68,3.82)$ & $<0.0001$ & $1.97(1.59,2.45)$ & $<0.0001$ \\
\hline$P$ for trend & $<0.0001$ & & $<0.0001$ & & $<0.0001$ & \\
\hline \multicolumn{7}{|c|}{ 90-day all-cause mortality } \\
\hline Anion gap & $1.09(1.07,1.10)$ & $<0.0001$ & $1.09(1.08,1.10)$ & $<0.0001$ & $1.04(1.02,1.06)$ & $<0.0001$ \\
\hline \multicolumn{7}{|c|}{ Anion gap (tertile) } \\
\hline$<13$ & 1 (ref) & & 1 (ref) & & 1 (ref) & \\
\hline$\geq 13,<16$ & $1.36(1.20,1.54)$ & $<0.0001$ & $1.33(1.17,1.51)$ & $<0.0001$ & $1.17(1.03,1.34)$ & 0.0170 \\
\hline$\geq 16$ & $2.22(1.98,2.50)$ & $<0.0001$ & $2.23(1.98,2.51)$ & $<0.0001$ & $1.53(1.32,1.77)$ & $<0.0001$ \\
\hline$P$ for trend & $<0.0001$ & & $<0.0001$ & & $<0.0001$ & \\
\hline \multicolumn{7}{|c|}{ Anion gap (quartile) } \\
\hline$<12$ & 1 (ref) & & 1 (ref) & & 1 (ref) & \\
\hline$\geq 12,<14$ & $1.33(1.14,1.56)$ & 0.0004 & $1.31(1.12,1.54)$ & 0.0008 & $1.17(0.99,1.37)$ & 0.0637 \\
\hline$\geq 14,<17$ & $1.57(1.35,1.82)$ & $<0.0001$ & $1.53(1.32,1.77)$ & $<0.0001$ & $1.35(1.15,1.57)$ & $<0.0001$ \\
\hline$\geq 17$ & $2.57(2.23,2.97)$ & $<0.0001$ & $2.62(2.27,3.02)$ & $<0.0001$ & $1.66(1.39,1.98)$ & $<0.0001$ \\
\hline$P$ for trend & $<0.0001$ & & $<0.0001$ & & $<0.0001$ & \\
\hline
\end{tabular}

Models were derived from Cox proportional hazards regression models. Crude model adjusted for none. Model I adjusted for age, gender, and ethnicity. Model II adjusted for age, gender, ethnicity, temperature, systolic blood pressure, diastolic blood pressure, respiratory rate, heart rate, percutaneous oxygen saturation, weight, atrial fibrillation, liver disease, valvular heart diseases, pulmonary circulation diseases, pneumonia, respiratory failure, diabetes, stroke, malignancy, lactate, prothrombin time, white blood cell, blood urea nitrogen, creatinine, potassium, bicarbonate, glucose, vasopressor, dialysis, mechanical ventilation, SOFA, SAPSII, and NT-proBNP. Note: HR: hazard ratio; CI: confidence interval; SOFA: stroke, and malignancy. Calculate the sequential organ failure assessment score; SAPSII: simplified acute physiology score II; NT-proBNP: N-terminal probrain natriuretic peptide.

95\% CI: $2.62,2.27-3.03 ; 2.23,1.98-2.51$; quartiles 4 versus quartiles 1: HR, 95\% CI: 3.20, 2.68-3.82; 2.62, 2.27-3.02). In model II, after adjusting for age, sex, ethnicity, temperature, $\mathrm{HR}, \mathrm{RR}, \mathrm{SBP}, \mathrm{DBP}, \mathrm{SpO}_{2}$, weight, atrial fibrillation, liver disease, valvular heart diseases, pulmonary circulation diseases, pneumonia, respiratory failure, diabetes, stroke, malignancy, SOFA, SAPSII, lactate, NT-proBNP, PT, WBC, BUN, creatinine, potassium, bicarbonate, glucose, vasopressor, dialysis, and mechanical ventilation, high levels of SAG were still an independent predictor of 30- and 90-day all-cause mortalities (tertile 3 versus tertile 1: HR, 95\% CI: 1.74, 1.46-2.08; 1.53, 1.32-1.77; quartiles 4 versus quartiles 1: $\mathrm{HR}, 95 \% \mathrm{CI}: 1.97$, $1.59-2.45 ; 1.66,1.39-1.98)$.

3.3. Secondary Outcome: Association between SAG and Readmission and MACEs. In this study, readmission rate and MACEs were regarded as the secondary outcome. Multivariate logistic regression indicated that increased SAG was associated with increased risk of MACEs in critically ill patients with $\mathrm{CHF}$ (tertile 3 versus tertile 1: OR, 95\% CI: $1.76,1.50-2.07)$. No obvious correlation between SAG and readmission rate was observed in critically ill patients with CHF (tertile 3 versus tertile 1: OR, 95\% CI: 1.05, 0.88-1.24). The detailed data are shown in Supplementary Table 1.

3.4. Subgroup Analyses. Subgroup analyses were employed to assess the association between SAG and 30-day all-cause mortality. No significant interactions were found in most strata, as shown in Table 4, except for pneumonia, malignancy, respiratory failure, and mechanical ventilation. Among patients with $\mathrm{CHF}$ and high SAG, those with a comorbidity of pneumonia had a significantly lower 30-day mortality risk ( $\mathrm{HR}, 95 \% \mathrm{CI}: 1.44,1.13-1.85$ versus 3.27 , 2.73-3.91). Similar trends also appeared in patients with a history of malignancy, respiratory failure, and mechanical ventilation (HR, 95\% CI: 1.84, 1.10-3.07 versus 2.71, 2.33- 
TABLE 4: Subgroup analysis of the correlation between serum anion gap and 30-day all-cause mortality in critically ill patients with congestive heart failure.

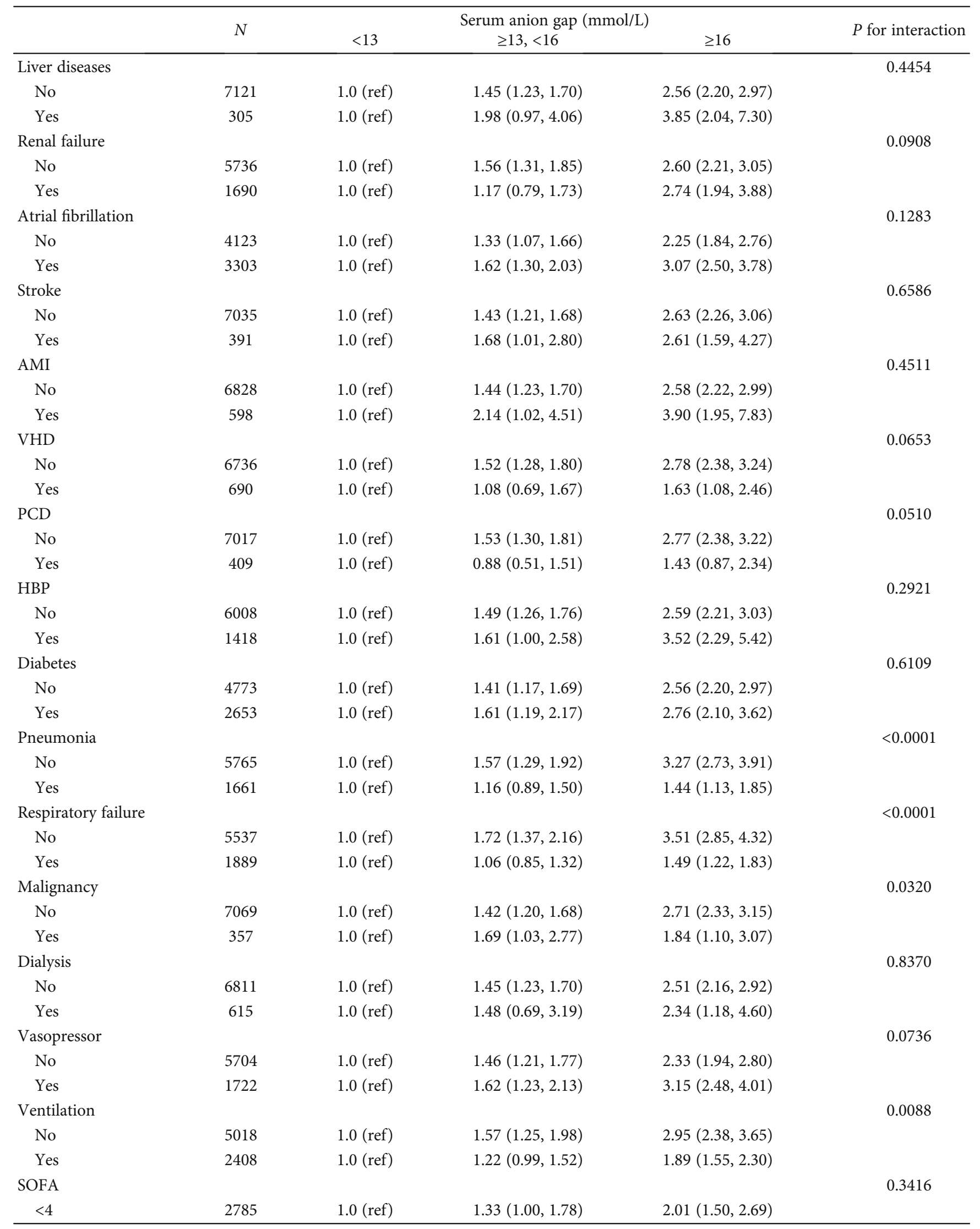


TABLE 4: Continued.

\begin{tabular}{|c|c|c|c|c|c|}
\hline \multirow{2}{*}{\multicolumn{2}{|c|}{$N$}} & \multicolumn{3}{|c|}{ Serum anion gap $(\mathrm{mmol} / \mathrm{L})$} & \multirow{2}{*}{$P$ for interaction } \\
\hline & & $<13$ & $\geq 13,<16$ & $\geq 16$ & \\
\hline$\geq 4$ & 4641 & 1.0 (ref) & $1.56(1.30,1.89)$ & $2.59(2.19,3.07)$ & \\
\hline SAPSII & & & & & 0.8125 \\
\hline$<39$ & 3701 & 1.0 (ref) & $1.50(1.13,2.00)$ & $2.17(1.62,2.91)$ & \\
\hline$\geq 39$ & 3725 & 1.0 (ref) & $1.36(1.12,1.64)$ & $2.09(1.77,2.48)$ & \\
\hline
\end{tabular}

Note: AMI: acute myocardial infarction; VHD: valvular heart diseases; PCD: pulmonary circulation diseases; HBP: hypertension; SOFA: stroke, and malignancy. Calculate the sequential organ failure assessment score; SAPSII: simplified acute physiology score II.

$3.15 ; 1.49,1.22-1.83$ versus $3.51,2.85-4.32 ; 1.89,1.55-2.30$ versus $2.95,2.38-3.65)$.

\section{Discussion}

CHF is a common critical illness in the field of cardiovascular disease, with characteristics of high prevalence, hospital admissions, readmissions, and even mortality [19]. According to the Framingham study, the 5-year survival rate of patients with $\mathrm{CHF}$ for men is only $25 \%$, whereas that for women is $38 \%$, which heavily threatens human health [20,21]. Unfortunately, a lack of clinical objective indicators to predict the prognosis of patients with CHF exists at present. Various laboratory tests, such as blood gas analysis and biochemical testing, are often clinically used to determine the status of the acid-base balance of patients, but the relationship between these indicators and the prognosis of patients with $\mathrm{CHF}$ is rarely studied. Therefore, finding a simple and effective laboratory parameter to predict the prognosis of patients with $\mathrm{CHF}$ is particularly important and valuable for clinical treatment.

In this study, the possible prognostic value of SAG in patients with CHF was explored. Increased SAG was associated with increased risk of 30- and 90-day all-cause mortalities, and it remained as an independent predictor for all-cause mortality in critically ill patients with $\mathrm{CHF}$ after adjusting for age, gender, ethnicity, and another confounder. Besides, no significant interactions were found between SAG and most covariables for 30-day mortality, thus enhancing the stability and consistency of the results. To the best of the author's knowledge, this research was the first to clarify that high levels of SAG were associated with poor prognosis of critically ill patients with $\mathrm{CHF}$.

As a routine examination that almost all admitted patients complete, SAG has the characteristics of simple calculation and easy acquisition, even without arterial puncture [14]. By retrospectively analyzing the electrolyte test results of 6868 hospitalized patients, Lolekha et al. [22] showed that there are SAG abnormalities in $40.5 \%$ of patients, including an increase in $37.6 \%$ of patients, and a decrease in $2.9 \%$. Moreover, a series of studies in recent years further indicated that SAG could be used as a new prognosis biomarker to stratify patients at risk of deterioration, and patients could benefit from further treatment [5]. Sahu and his colleagues [23] reported that SAG acidosis was independently associated with inhospital all-cause mortality in patients with AMI (OR,
95\% CI: 4.2, 2.3-7.5), with longer average length of stay (5.1 versus 3.3 days). In critical ill patients with aortic aneurysm, Chen et al. [14] also demonstrated that SAG could effectively predict ICU mortality, and the risk of ICU death could increase by $38 \%$ for every increase of $1 \mathrm{mEq} / \mathrm{L}$ in SAG. Regrettably, the specific mechanism of $\mathrm{SAG}$ as a prognostic biomarker remains unclear.

The relationship between SAG and 30-day all-cause mortality in critically ill patients with CHF presented a U-shaped curve, which showed an elevated mortality risk at decreased and increased SAGs. In clinical practice, a decrease in SAG is usually rare, which may be related to the decrease in untested anions (such as hypoalbuminemia) or even laboratory error [24]. Albumin plays a remarkable role in various physiological processes, including maintaining colloidal osmotic pressure and microvascular integrity, ligand binding and material transport, antioxidant and antithrombotic effects, and enzyme activities [25]. Low level of blood albumin could promote and aggravate circulatory congestion and strengthen oxidative stress, inflammatory response, and susceptibility to infection, which could worsen the prognosis of patients with CHF [26]. In patients with acute heart failure, hypoalbuminemia has been proven to be associated with increased hospital mortality, and it served as an independent predictor of long-term mortality [27]. The increase in SAG is relatively more common, and previous studies have illustrated that the accumulation of serum lactate and ketone body accounted for $62 \%$ of the cause of the increase in SAG [28]. In CHF, the heart loses its ability to efficiently pump blood, which leads to decreased perfusion of tissues and hypoxia of cells [29]. Under anaerobic conditions, glucose undergoes glycolysis and eventually generates lactate, which may be primarily responsible for the increased SAG in patients with $\mathrm{CHF}$ [30]. In addition, sympathetic excitation in CHF contributes to the overproduction of lactate [31]. Under normal condition, the production and clearance of lactate are in balance, and this clearance is mainly responsible for the liver and kidney. Patients with CHF are often complicated by liver and kidney dysfunction, which further aggravates hyperlactataemia and the increase in SAG [32]. In the present study, the relationship between lactate and 30-day all-cause mortality in critically ill patients with CHF was also determined. Consistent with the results of previous studies [33], the increase in lactate was significantly correlated with poor prognosis (HR, 95\% CI: 1.73, 1.53-1.95), which may be related to the damage and dysfunction of organs caused 
by tissue hypoperfusion and neurohormonal abnormalities. After adjusting for covariates including lactate, the anion gap was still associated with poor prognosis, thus showing the unique value of SAG as an independent predictor of the prognosis of critically ill patients with CHF.

This research has some limitations. First, it was a singlecenter study, whose subjects were relatively seriously ill. The results may not be applicable to all patients with $\mathrm{CHF}$. Second, some unknown or even vital risk factors were not considered, although two multivariate models were used to control the influence of confounder on the outcome variables. Third, the SAG concentration of patients was only used when they entered the ICU to assess the relationship between them and all-cause mortality. It may be more valuable for the prognosis prediction if SAG can be dynamically monitored. Fourth, due to the lack of records of albumin, we did not perform albumin correction on the SAG, although some studies have shown that hypoalbuminemia could affect the SAG concentration [34]. Finally, the accuracy and efficiency of NT-proBNP and SAG in predicting the poor prognosis of critically ill patients with CHF were not compared, hence the possible exclusion bias.

\section{Conclusion}

SAG can effectively predict the 30- and 90-day all-cause mortalities of critically ill patients with CHF. It is expected to become a simple and effective marker for prognostic evaluation in these patients. Monitoring of SAG concentration could clinically help identify high-risk patients early and choose a more scientific treatment plan for patients.

\section{Data Availability}

Publicly available datasets were analyzed in this study. This data can be extracted from Monitoring in Intensive Care Database III version 1.4 (MIMIC-III v.1.4) after passing on the required courses and obtaining the authorization.

\section{Conflicts of Interest}

All authors declare that there is no conflict of interest.

\section{Authors' Contributions}

Author Z. X. Y designed the research. Y.Y.T performed the data analysis and drafted the manuscript. W.C.L, M.Q, G.J.L, L.H.Z, X.F.Z, and Z.H.L analyzed the data and revised the manuscript.

\section{Acknowledgments}

Our study was supported by the National Natural Science Foundation of China (81873416) and the National Science and Technology Major Project (2017ZX0930401405).

\section{Supplementary Materials}

Supplementary Table 1: ORs (95\% CIs) for MACEs and readmission across groups of serum anion gap. (Supplementary Materials)

\section{References}

[1] D. Sun, F. Zhang, T. Ma, Y. Zhang, and Z. Liang, "Atorvastatin alleviates left ventricular remodeling in isoproterenol-induced chronic heart failure in rats by regulating the RhoA/Rho kinase signaling pathway," Pharmacological Reports, vol. 72, no. 4, pp. 903-911, 2020.

[2] F. Orso, G. Fabbri, and A. P. Maggioni, "Epidemiology of heart failure," Handbook of Experimental Pharmacology, vol. 243, pp. 15-33, 2017.

[3] A. J. Peixoto and R. J. Alpern, "Treatment of severe metabolic alkalosis in a patient with congestive heart failure," American Journal of Kidney Diseases, vol. 61, no. 5, pp. 822-827, 2013.

[4] J. J. Park, D. J. Choi, C. H. Yoon et al., "The prognostic value of arterial blood gas analysis in high-risk acute heart failure patients: an analysis of the Korean Heart Failure (KorHF) registry," European Journal of Heart Failure, vol. 17, no. 6, pp. 601-611, 2015.

[5] S. A. Glasmacher and W. Stones, "Anion gap as a prognostic tool for risk stratification in critically ill patients - a systematic review and meta-analysis," BMC Anesthesiology, vol. 16, no. 1, p. $68,2016$.

[6] S. B. Lee, D. H. Kim, T. Kim et al., "Anion gap and base deficit are predictors of mortality in acute pesticide poisoning," Human \& Experimental Toxicology, vol. 38, no. 2, pp. 185192, 2019.

[7] B. Mitra, C. Roman, K. E. Charters, G. O'Reilly, D. Gantner, and P. A. Cameron, "Lactate, bicarbonate and anion gap for evaluation of patients presenting with sepsis to the emergency department: a prospective cohort study," Emergency Medicine Australasia, vol. 32, no. 1, pp. 20-24, 2019.

[8] B. Cheng, D. Li, Y. Gong, B. Ying, and B. Wang, "Serum anion gap predicts all-cause mortality in critically ill patients with acute kidney injury: analysis of the MIMIC-III database," Disease Markers, vol. 2020, Article ID 6501272, 10 pages, 2020.

[9] T. Banerjee, D. C. Crews, D. E. Wesson et al., "Elevated serum anion gap in adults with moderate chronic kidney disease increases risk for progression to end-stage renal disease," American Journal of Physiology. Renal Physiology, vol. 316, no. 6, pp. F1244-F1253, 2019.

[10] J. J. Leskovan, C. F. Justiniano, J. A. Bach et al., “Anion gap as a predictor of trauma outcomes in the older trauma population: correlations with injury severity and mortality," The American Surgeon, vol. 79, no. 11, pp. 1203-1206, 2013.

[11] S. W. Yang, Y. J. Zhou, Y. X. Zhao et al., "The serum anion gap is associated with disease severity and all-cause mortality in coronary artery disease," Journal of Geriatric Cardiology, vol. 14, no. 6, pp. 392-400, 2017.

[12] A. E. Johnson, T. J. Pollard, L. Shen et al., "MIMIC-III, a freely accessible critical care database," Scientific Data, vol. 3, no. 1, p. 160035, 2016.

[13] W. Li, A. Huang, H. Zhu et al., "Gut microbiota-derived trimethylamine $\mathrm{N}$-oxide is associated with poor prognosis in patients with heart failure," The Medical Journal of Australia, vol. 213 , no. 8, pp. 374-379, 2020. 
[14] Q. Chen, Q. Chen, L. Li et al., "Serum anion gap on admission predicts intensive care unit mortality in patients with aortic aneurysm," Experimental and Therapeutic Medicine, vol. 16, no. 3, pp. 1766-1777, 2018.

[15] J. Allard, S. Cotin, F. Faure et al., "SOFA-an open source framework for medical simulation," Studies in Health Technology and Informatics, vol. 125, pp. 13-18, 2007.

[16] J. R. Le Gall, S. Lemeshow, and F. Saulnier, "A new simplified acute physiology score (SAPS II) based on a European/North American multicenter study," JAMA, vol. 270, no. 24, pp. 2957-2963, 1993.

[17] M. Feng, J. I. McSparron, D. T. Kien et al., "Transthoracic echocardiography and mortality in sepsis: analysis of the MIMIC-III database," Intensive Care Medicine, vol. 44, no. 6, pp. 884-892, 2018.

[18] V. W. Jaddoe, L. L. de Jonge, A. Hofman, O. H. Franco, E. A. Steegers, and R. Gaillard, "First trimester fetal growth restriction and cardiovascular risk factors in school age children: population based cohort study," BMJ, vol. 348, no. jan23 1, p. g14, 2014.

[19] Q. Ma, Y. Luo, P. Guo et al., "Clinical effects of Xinmailong therapy in patients with chronic heart failure," International Journal of Medical Sciences, vol. 10, no. 5, pp. 624-633, 2013.

[20] S. Stewart, K. MacIntyre, D. J. Hole, S. Capewell, and J. J. McMurray, "More 'malignant' than cancer? Five-year survival following a first admission for heart failure," European Journal of Heart Failure, vol. 3, no. 3, pp. 315-322, 2001.

[21] Q. Wang, L. Dong, Z. Jian, and X. Tang, "Effectiveness of a PRECEDE-based education intervention on quality of life in elderly patients with chronic heart failure," BMC Cardiovascular Disorders, vol. 17, no. 1, p. 262, 2017.

[22] P. H. Lolekha, S. Vanavanan, and S. Lolekha, "Update on value of the anion gap in clinical diagnosis and laboratory evaluation," Clinica Chimica Acta, vol. 307, no. 1-2, pp. 33-36, 2001.

[23] A. Sahu, H. A. Cooper, and J. A. Panza, "The initial anion gap is a predictor of mortality in acute myocardial infarction," Coronary Artery Disease, vol. 17, no. 5, pp. 409-412, 2006.

[24] G. Dominguez-Cherit and S. A. Namendys-Silva, "Changes in the anion gap," Critical Care Medicine, vol. 41, no. 1, pp. 336$337,2013$.

[25] G. J. Quinlan, G. S. Martin, and T. W. Evans, "Albumin: biochemical properties and therapeutic potential," Hepatology, vol. 41, no. 6, pp. 1211-1219, 2005.

[26] I. Gotsman, A. Shauer, D. R. Zwas, I. Tahiroglu, C. Lotan, and A. Keren, "Low serum albumin: a significant predictor of reduced survival in patients with chronic heart failure," Clinical Cardiology, vol. 42, no. 3, pp. 365-372, 2019.

[27] J. L. Bonilla-Palomas, A. L. Gamez-Lopez, M. Moreno-Conde et al., "Hypoalbuminemia in acute heart failure patients: causes and its impact on hospital and long-term mortality," Journal of Cardiac Failure, vol. 20, no. 5, pp. 350-358, 2014.

[28] P. A. Gabow, W. D. Kaehny, P. V. Fennessey, S. I. Goodman, P. A. Gross, and R. W. Schrier, "Diagnostic importance of an increased serum anion gap," The New England Journal of Medicine, vol. 303, no. 15, pp. 854-858, 1980.

[29] M. S. Ali Sheikh, U. Salma, B. Zhang, J. Chen, J. Zhuang, and Z. Ping, "Diagnostic, prognostic, and therapeutic value of circulating miRNAs in heart failure patients associated with oxidative stress," Oxidative Medicine and Cellular Longevity, vol. 2016, Article ID 5893064, 13 pages, 2016.
[30] M. Fulop, M. Horowitz, A. Aberman, and E. R. Jaffe, "Lactic acidosis in pulmonary edema due to left ventricular failure," Annals of Internal Medicine, vol. 79, no. 2, pp. 180-186, 1973.

[31] R. Zymlinski, J. Biegus, M. Sokolski et al., "Increased blood lactate is prevalent and identifies poor prognosis in patients with acute heart failure without overt peripheral hypoperfusion," European Journal of Heart Failure, vol. 20, no. 6, pp. 10111018, 2018.

[32] S. Orn and G. van Hall, "Does a normal peripheral lactate value always indicate an aerobic tissue metabolism?," European Journal of Heart Failure, vol. 19, no. 8, pp. 1034-1035, 2017.

[33] T. Kawase, M. Toyofuku, T. Higashihara et al., "Validation of lactate level as a predictor of early mortality in acute decompensated heart failure patients who entered intensive care unit," Journal of Cardiology, vol. 65, no. 2, pp. 164-170, 2015.

[34] Y. Kotake, "Unmeasured anions and mortality in critically ill patients in 2016," Journal of Intensive Care, vol. 4, no. 1, p. $45,2016$. 